\title{
FRECUENCIA Y FACTORES ASOCIADOS AL DOLOR DE CUELLOO Y ESPALDA EN LOS ESTUDIANTES DE VI A X SEMESTRES DE LAS CLINICAS ODONTOLOGICAS DE LA UNIVERSIDAD SANTO TOMÁS
}

${ }^{1}$ Alix R. Figueredo R., ' Suleyma Martínez B., ${ }^{1}$ Natividad Rodríguez H., ${ }^{1}$ Gabriela Rueda M., ${ }^{2}$ Diana M. Camargo L., ${ }^{3}$ Miguel Ángel Guzmán T.

${ }^{1}$ Estudiante de X semestre, Facultad de Odontología, U. Santo Tomás, ${ }^{2}$ M Sc Epidemiología, Prof. E. Fisioterapia, U. Industrial de Santander, ${ }^{3}$ Médico General, Docente U. Santo Tomás

Autor responsable de correspondencia: Dr. Miguel Ángel Guzmán T.

Correo electrónico: miguelagt@hotmail.com

\begin{abstract}
RESUMEN
Objetivo: Determinar la frecuencia y los factores asociados al dolor de cuello y espalda en los estudiantes de VI a X semestre, de las clínicas odontológicas de la USTA.

Materiales y métodos: Se realizó un estudio observacional descriptivo. Los datos se obtuvieron de una encuesta realizada a la totalidad de los estudiantes de VI a X semestre de la facultad de odontología (112 estudiantes), durante el segundo período de 2002 y el primer período de 2003.

Resultados: Se estableció que la frecuencia de dolor lumbar fue del $22 \%$ y el dolor de cuello del $15.5 \%$ antes del ingreso a la clínica; en la actualidad, el dolor lumbar fue del 67.8\% y el dolor de cuello del 52.2\%. Esto demostró un aumento en la frecuencia de estas alteraciones con el ejercicio de la práctica odontológica, en total y por semestre $(\mathrm{p}<0.05)$. Se encontró que, el estrés es un factor asociado en forma estadísticamente significativa con la frecuencia del dolor lumbar y de cuello, en la población estudiada. Los factores de edad, comodidad de la silla y posturas inadecuadas, no se encontraron asociados. Conclusiones: Es importante promover la formulación y ejecución de programas de promoción de hábitos posturales saludables, entre los estudiantes de odontología y, a la vez, programas de prevención primaria de alteraciones musculoesqueléticas, derivadas de la práctica clínica odontológica, dirigidos a disminuir las alteraciones de cuello y espalda en los estudiantes y futuros profesionales de odontología. [Figueredo AR, Martínez S, Rodríguez N, Rueda G, Camargo DM, Guzmán MA. Frecuencia y factores asociados al dolor de cuello y espalda en los estudiantes de VI a X semestre de las clínicas odontológicas de la Universidad Santo Tomás. Ustasalud Odontología 2004; 3: 41 - 47]
\end{abstract}

Palabras clave: Estrés, Ergonomía, Dolor lumbar, Dolor de cuello.

\section{FREQUENCY AND RELATED FACTORS TO NECK AND BACK PAIN IN THE STUDENTS FROM SIXTH TO TENTH SEMESTER OF THE DENTAL CLINICS FROM THE SANTO TOMAS UNIVERSITY}

\begin{abstract}
Purpose: To establish the frequency and related factors to neck pain and back pain in the students who have been working in the dentistry clinics from the Santo Tomas University, by mean of a descriptive and observational study.

Material and methods: The information was collected through a test, which was made to the whole population of students from sixth to tenth semester of the dentistry faculty (112 students), in the 2002 second period and 2003 first period. Results: It was established that, frequency of back pain was $22 \%$ and neck pain $15.5 \%$ in the students before they were be admitted to clinics, and after that, frequency of back pain was $67.8 \%$ and neck pain $52.2 \%$. It shows a clear increase in the frequency of these complaints with the beginning of the dentistry clinical practice. It was found that, stress is a statistically significative factor with the presentation of back and neck pain in this population. Other factors such as age, chair comfort and bad postures, weren't statistically significative. The later is in contradiction with others studies that established them like strong related factors to such alterations.

Conclusions: It's a very important point, to create and execute promotion and prevention programs to healthy postural habits in dentistry students, to avoid inadequate postures and musculoeskeletal disorders and guided to decrease the back and neck alterations in the students and so, in the future professionals of dentistry.
\end{abstract}

Key words: Stress, Ergonomics, Lumbago, Neck pain.

Recibido para publicación: 10 de marzo de 2004. Aceptado para publicación: 30 de mayo de 2004. 


\section{INTRODUCCIÓN}

La asociación Internacional del estudio del dolor (IASP) lo define como una experiencia sensorial desagradable con daño tisular actual o potencial o descrito en términos de dicho daño.

Uno de los motivos más frecuentes de la consulta por dolor del músculo esquelético es el síndrome del dolor lumbar,2 definido como una entidad clínica caracterizada por una alteración estructural o una sobrecarga funcional-postural de los elementos, que forman la columna lumbar.3 En la mayoría de los casos el dolor lumbar cede con el reposo y se exacerba con el movimiento; es decir, es un dolor típicamente mecánico. ${ }^{2}$

El dolor en el cuello constituye la segunda incapacidad músculo-esquelética más importante. Su unidad funcional es idéntica al segmento de la columna lumbar, excepto que se desliza en una dirección ántero posterior. ${ }^{3}$

Unas posturas inadecuadas de trabajo del odontólogo, mantenidas en forma reiterada, pueden dar lugar a patologías del sistema músculo-esquelético, siendo las afecciones de la columna vertebral (dolor lumbar, dolor del cuello) las patologías más frecuentes. ${ }^{4}$

La Organización Mundial de la Salud define el trastorno de origen laboral el producido por una serie de factores. El entorno laboral y la realización del trabajo contribuyen significativamente, aunque no siempre en la misma medida. Son los que desencadenan la enfermedad. Algunos de los trastornos clasificados como músculoesqueléticos son los trastornos degenerativos de la columna, que afectan habitualmente al cuello y la región lumbar. $^{5}$

En la literatura revisada, de nivel internacional, se encontró que los odontólogos y auxiliares dentales presentaron mayor prevalencia del dolor lumbar y de cuello, debido al estrés, la tensión y las posturas inadecuadas. Así consta en un estudio realizado por el Dr. Alwassan en la ciudad de Riyadh, Arabia Saudita. En él se demostró que 111 de los sujetos examinados sufrieron dolor de cuello y 150 (73.5\%), dolor de espalda. ${ }^{6}$

En otras revisiones se halló que la incidencia de los desórdenes músculo-esqueléticos eran muy pocos; pero en Nebraska, el 29\% de más de mil odontólogos relataron síntomas de neuropatías en los miembros superiores o el cuello. Estos desórdenes músculo-esqueléticos se relacionaron con los movimientos repetidos de los miembros superiores y las posturas prolongadas durante las actividades, comunes en la odontología.?

En la literatura revisada de nivel nacional no se hallaron referencias basadas en la frecuencia de dolor de cuello y espalda, en odontólogos. Sin embargo, en la encuesta nacional de dolor ACED 2002, se reportó que el 50\% de la población colombiana presenta algún tipo de dolor. De este porcentaje, cerca del $8 \%$ se relaciona con molestias en la espalda.

El objetivo de esta investigación es determinar la frecuencia y los factores asociados al dolor de cuello y espalda, en los estudiantes de VI a X semestres, de las clínicas odontológicas de la Universidad Santo Tomás, y a la vez, proponer programas de promoción de hábitos saludables posturales para estudiantes y profesionales de odontología, en general, así como programas de prevención primaria y secundaria.

\section{MATERIALES Y MÉTODOS}

Se aplico un estudio de tipo observacional descriptivo, cuya población estuvo conformada por la totalidad de alumnos de VI a X semestres, de la facultad de odontología, durante el primer período del 2003 (112 estudiantes, en total). Se excluyeron del estudio los estudiantes de semestres que no realizan sus prácticas clínicas.

En esta investigación se aplicó un instrumento tipo encuesta, donde se registraron variables sociodemográficas como edad, género y semestre; antecedentes médicos como medición del dolor para establecer su ausencia o presencia del mismo, antes de entrar a las prácticas clínicas, y posibles factores asociados al dolor de cuello y espalda.

De igual manera se aplicó un formulario Oswestry para el dolor de cuello y espalda. ${ }^{8}$ Ambos cuestionarios se aplicaron para evaluar la limitación funcional en las actividades de la vida diaria, secundarias al dolor de cuello y de espalda. Estos cuestionarios se encuentran divididos en nueve atributos funcionales, además de la intensidad del dolor, equivalentes a la alteración funcional producida por el dolor de cuello y espalda. Se manejan en una escala de categorías ordenadas de seis puntos, que van de 0 a 5, desde ausencia de dolor a máxima alteración funcional secundaria ante la presencia de dolor de cuello y espalda. ${ }^{8}$ 


\section{Análisis}

Inicialmente se describió la población de estudio en sus características sociodemográficas y antecedentes médicos. Se estableció la frecuencia de dolor en la población de estudio y las alteraciones funcionales subsiguientes a la experiencia dolorosa, mediante tablas de frecuencia. Posteriormente se evaluaron las posibles asociaciones entre las variables sociodemográficas con los resultados del formulario Oswestry, aplicando una prueba de $\mathrm{X}^{2}$ con un nivel de significancía $\alpha=0.05 .{ }^{9}$ Para el análisis de la evolución del dolor y comparar la presencia de dolor antes y después de ingresar a la clínica, se utilizó $\mathrm{X}^{2} \mathrm{Mc}$ Nemar. La base de datos se elaboró en Excel ${ }^{10}$ y el análisis en STATA 6.0. ${ }^{11}$

\section{Consideraciones éticas}

Según el artículo 11 de la Resolución 008430, de 1993, del Ministerio de Salud de Colombia, este trabajo se consideró una investigación sin riesgo, ya que se realizó con técnicas y métodos sin ninguna intervención, que modificara las variables biológicas, fisiológicas, psicológicas o sociales de los individuos que participaron en el mismo

\section{RESULTADOS}

\section{Descripción general}

En total, fueron encuestados 112 estudiantes, 87 (77.68\%) del género femenino, con un promedio de edad de 22 años y un rango entre 18 y 30 años. La distribución por semestre fue equitativa a excepción de sexto semestre que presentó la mayor frecuencia (26.8\%); de séptimo a décimo semestres, entre $17 \%$ a $18 \%$ para cada semestre respectivamente (Tabla 1).

\section{SEMESTRE FRECUENCIA PORCENTAJE}

\begin{tabular}{ccc}
\hline 6 & 30 & 26.79 \\
7 & 19 & 16.96 \\
8 & 21 & 18.75 \\
9 & 21 & 18.75 \\
10 & 21 & 18.75 \\
\hline$n=112$ & &
\end{tabular}

$n=112$

Tabla 1. Distribución de estudiantes por semestre.

\section{Antecedentes Médicos}

Información relacionada con dolor de espalda y cuello: Al explorar la variable de dolor, se encontró que 74 estudiantes (66.1\%) presentan dolor de espalda en la actualidad, y 57 estudiantes (51.4\%) presentan dolor de cuello.

Antes de entrar a la clínica tan sólo presentaron dolor de espalda 24 (22\%) y dolor de cuello 17 (15.6\%). Consultaron al médico por dolor de espalda 24 (22.02\%), y 17 (15.60\%) por dolor de cuello. Se observó que 14 (12.96\%) recibieron tratamiento fisioterapéutico para el dolor de espalda, y 10 (9.26\%) recibieron tratamiento para dolor de cuello. Un $93 \%$ de los estudiantes considera necesario recibir educación relacionada con la postura adecuada en el puesto de trabajo.

Posibles causas: Aunque las causas pueden ser diferentes y lo que cada persona crea como generador de su dolor, al analizar las posibles causas del dolor se encontró que la comodidad de la silla para realizar los procedimientos odontológicos, sólo fue mencionada por 29 estudiantes 10 que equivale al $26.7 \%$.

Entre los factores que los estudiantes asocian más frecuentemente con el dolor se encuentran: la intensidad horaria de trabajo, que influye en el dolor de espalda (71.3\%) y de cuello (57.0\%); y el estrés, asociado con el dolor de espalda $(86,1 \%)$ y de cuello (78.4\%). En la tabla 2 se observa la distribución de las causas para el dolor de cuello y espalda en lo estudiantes.

Descripción de la alteración funcional en la espalda: En las alteraciones funcionales generadas por el dolor de espalda, al aplicar el formulario Oswestry, se halló que el estar de pie (64.8\%), los viajes (59.4\%) y el cuidado personal (63.5\%), elevaban la intensidad de dolor (47.3\%), en la categoría de moderado. Se refleja en una categoría, de moderado a severo nuevamente, al estar de pie (17.5\%) y levantar objetos (12.1\%). Los resultados, al aplicar el formulario de dolor lumbar de Oswestry, se presentan en la tabla 3.

Descripción de la alteración funcional en el cuello: Las alteraciones funcionales generadas por el dolor del cuello, al aplicar el formulario Oswestry, se encontró que la lectura era más alta en la categoría moderada (57.8\%), intensidad de dolor (54.3\%), recreación (49.1\%), concentración (42.1\%). Se refleja en la categoría de moderado a severo, el dolor de cabeza (59.6\%), y nuevamente la lectura (28.0\%). Los resultados al aplicar el formulario Oswestry de dolor de cuello se presentan en la tabla 4. 


\begin{tabular}{lcc}
\hline POSIBLES CAUSAS & NÚMERO & PORCENTAJE \\
\hline Comodidad de la silla odontológica & 29 & 26.7 \\
Posibilidad de variación de la postura & 68 & 63.0 \\
Posibilidad para alcanzar instrumentos & 67 & 62.0 \\
Intensidad horaria en dolor de espalda & 77 & 71,3 \\
Intensidad horaria en dolor de cuello & 61 & 57,0 \\
Posibilidad de ejercer actividades diarias & 9 & 8,30 \\
Influencia del estrés en el dolor de espalda & 93 & 86,1 \\
Influencia del estrés en el dolor de cuello & 85 & 79,4 \\
\hline
\end{tabular}

Tabla 2. Distribución de las causas de dolor de cuello y de espalda en los estudiantes.

El análisis bivariado en dolor lumbar, considerados los posibles factores asociados, se presentan en la tabla 5. No se encontraron asociaciones estadísticamente significativas por género, edad y semestre con el nivel de discapacidad establecida. Al analizar las posibles causas asociadas con el dolor lumbar se encontró que la comodidad de la silla y de los objetos alcanzables se encuentran asociados con el nivel de discapacidad leve.
El análisis bivariado del dolor de cuello se muestra, en forma similar, al de dolor lumbar. No hubo asociación estadísticamente significativa entre las variables de género, edad y semestre con el nivel de discapacidad de cuello (Tabla 6). Al analizar las posibles causas se encontró una asociación estadísticamente significativa entre el tiempo laboral y el estrés con el aumento del nivel de discapacidad ( $\mathrm{p}=0.001, \mathrm{p}=0.042)$, respectivamente.

\begin{tabular}{lccc}
\hline ALTERACión FUNCIONAL & AUSENTE A LEVE & MODERADO & MODERADO A SEVERO \\
\hline Intensidad del dolor & $28(37.8 \%)$ & $35(47.3 \%)$ & $5(6.7 \%)$ \\
Cuidado personal & $21(28.3 \%)$ & $47(63.5 \%)$ & $6(8.1 \%)$ \\
Levantar objetos & $34(46 \%)$ & $31(41.8 \%)$ & $9(12.1 \%)$ \\
Desplazamientos & $61(82.4 \%)$ & $11(14.8 \%)$ & $2(2.7 \%)$ \\
Sentarse & $42(56.7 \%)$ & $15(20.2 \%)$ & $17(23 \%)$ \\
Estar de pie & $13(17.5 \%)$ & $48(64.8 \%)$ & $13(17.5 \%)$ \\
Dormir & $63(85.196)$ & $10(13.5 \% 1)$ & $1(1.35 \%)$ \\
Vida sexual & $60(81.0 \%)$ & $12(16.2 \%)$ & $2(2.7 \%)$ \\
Vida social & $40(54.0 \%)$ & $29(39.1 \%)$ & $5(6.7 \%)$ \\
Viajes & $22(29.7 \%)$ & $44(59.4 \%)$ & $8(10.8 \%)$ \\
\hline
\end{tabular}

Tabla 3. Descripción de la alteración funcional según las categorías de dolor lumbar. 


\begin{tabular}{lccc}
\hline ALTERACión FUNCIONAL. & AUSENTE A LEVE & MODERADO & MODERADO A SEVERO \\
\hline Intensidad del dolor & $11(19.3 \%)$ & $31(54.3 \%)$ & $15(26.3 \%)$ \\
Cuidado personal & $31(54.3 \%)$ & $22(38.6 \%)$ & $4(7.0 \%)$ \\
Levantar objetos & $28(49.1 \%)$ & $23(40.3 \%)$ & $6(10.5 \%)$ \\
Lectura & $8(14.0 \%)$ & $33(57.8 \%)$ & $16(28.0 \%)$ \\
Dolores de cabeza & $7(12.2 \%)$ & $16(28.0 \%)$ & $34(59.6 \%)$ \\
Concentración & $21(36.8 \%)$ & $24(42.1 \%)$ & $12(21.0 \%)$ \\
Trabajo & $32(56.1 \%)$ & $19(33.3 \%)$ & $6(10.5 \%)$ \\
Conduciendo & $30(52.6 \%)$ & $21(36.8 \%)$ & $6(10.5 \%)$ \\
Durmiendo & $31(54.3 \%)$ & $18(31.5 \%)$ & $8(14.0 \%)$ \\
Recreación & $24(42.1 \%)$ & $28(49.1 \%)$ & $5(8.7 \%)$ \\
\hline
\end{tabular}

Tabla 4. Descripción de la alteración funcional según las categorías de dolor del cuello.

Cabe destacar que, a pesar de ser estadísticamente significativo, los estudiantes manifiestan que no encuentran dificultad para trabajar, a pesar del nivel de discapacidad.

Al analizar si el ingreso a la clínica aumentaba o no, en forma relevante, la presencia del dolor de cuello y espal$\mathrm{da}$, se encontró que la frecuencia de ambas alteraciones se incrementa de una forma significativa en cada semestre, si se comparan los porcentajes de dolor antes y después de ingresar a la clínica $(\mathrm{p}=<0.0001)$ (Tabla 7).

\section{DISCUSIÓN}

El dolor lumbar y el dolor de cuello son dos alteraciones fundamentales, que afectan a los odontólogos; es un problema de gran importancia dentro de la vida laboral del profesional de odontología, ya que afecta el rendimiento y calidad de su trabajo. ${ }^{6}$

Esta investigación determinó la frecuencia de dolor lumbar en un $22 \%$ y de dolor de cuello en un $15.5 \%$ de los estudiantes, antes de ingresar a la clínica. El dolor registrado en la actualidad correspondió, para dolor lumbar a un $67.8 \%$ y para dolor del cuello, a un $52.2 \%$ en los estudiantes de odontología. Estas cifras muestran con claridad que la frecuencia de dolor lumbar y de dolor de cuello

\begin{tabular}{|c|c|c|c|}
\hline Variables & $\begin{array}{c}\text { Leve } \\
\mathrm{n}=101\end{array}$ & $\begin{array}{l}\text { Moderada } \\
\qquad \mathbf{n}=11\end{array}$ & $\mathbf{p}$ \\
\hline Género femenino & 78 & 9 & 0.73 \\
\hline Edad $>22$ años & 45 & 4 & 0.603 \\
\hline \multicolumn{4}{|l|}{ Semestre } \\
\hline 6 & 27 & 3 & 0.545 \\
\hline 7 & 18 & 1 & \\
\hline 8 & 20 & 1 & \\
\hline 9 & 19 & 2 & \\
\hline 10 & 17 & 4 & \\
\hline \multicolumn{4}{|l|}{ Posibles causas } \\
\hline Comodidad de la silla & 29 & 0 & 0.034 \\
\hline Variación de la postura & 61 & 7 & 0.961 \\
\hline Objetos al alcance & 65 & 4 & 0.045 \\
\hline $\begin{array}{l}\text { Tiempo laboral } \\
\text { Horas laborales }\end{array}$ & 66 & 9 & 0.347 \\
\hline $0-3$ & 2 & 1 & \\
\hline $3-6$ & 82 & 9 & 0.37 \\
\hline$>6$ & 14 & 1 & \\
\hline Dificultad para trabajar & 5 & 4 & 0.0001 \\
\hline $\begin{array}{l}\text { El estrés contribuye al } \\
\text { dolor }\end{array}$ & 81 & 11 & 0.144 \\
\hline
\end{tabular}

Tabla 5. Evaluación de las posibles asociaciones entre variables sociodemográficas relacionadas con el dolor lumbar. 


\begin{tabular}{lcccc}
\hline \multicolumn{1}{c}{ Variables } & $\begin{array}{c}\text { Leve } \\
\mathrm{n}=93\end{array}$ & $\begin{array}{c}\text { Moderada } \\
\mathrm{n}=\mathbf{1 7}\end{array}$ & $\begin{array}{c}\text { Severa } \\
\mathrm{n}=\mathbf{2}\end{array}$ & $\mathbf{p}$ \\
\hline Género femenino & 70 & 15 & 2 & 0.37 \\
Edad $>22$ años & 42 & 5 & 2 & 0.13 \\
Semestre & & & & \\
6 & 27 & 2 & 1 & \\
7 & 18 & 1 & 0 & \\
8 & 16 & 5 & 0 & 0.26 \\
9 & 18 & 3 & 0 & \\
10 & 14 & 6 & 1 &
\end{tabular}

\begin{tabular}{lcccc} 
Posibles causas & & & & \\
Comodidad de la silla & 27 & 2 & 0 & 0.196 \\
Variación de la postura & 58 & 9 & 1 & 0.59 \\
Objetos al alcance & 58 & 10 & 1 & 0.81 \\
Tiempo laboral & 43 & 16 & 2 & 0.001 \\
Horas laborales & & & & \\
$0-3$ & 2 & 1 & 0 & \\
$3-6$ & 75 & 14 & 2 & 0.882 \\
$>6$ & 13 & 2 & 0 & \\
$\begin{array}{l}\text { Dificultad para trabajar } \\
\begin{array}{l}\text { El estrés contribuye al } \\
\text { dolor }\end{array}\end{array}$ & 65 & 2 & 1 & 0.078 \\
\hline
\end{tabular}

Tabla 6. Evaluación de las posibles asociaciones entre las variables sociodemográficas y las posibles causas relacionadas con el dolor de cuello.

se incrementa con el ejercicio de la práctica demostró una diferencia estadísticamente significativa en los factores asociados al dolor lumbar y dolor de cuello $(\mathrm{p}=$ $<0.0001$ ); esta diferencia fue constante en todos los semestres analizados.

Comparadas estas cifras con el estudio realizado por Alwazzan y colaboradores, en 2001, se encontraron frecuencias similares en dolor lumbar (73.5\%) y en dolor del cuello (54.4\%) en la población de odontólogos. Es importante resaltar que aunque la población de estudio es diferente, la frecuencia en dolor lumbar y dolor de cuello son muy similares.

Un estudio realizado con más de 3500 odontólogos registró que el $38 \%$ se encuentra frecuentemente ansioso 0 preocupado; el 34\% respondió que siempre o frecuente- mente se encuentra con cansancio físico o emocional, y el $26 \%$ presentó dolores de cabeza y dolores de espalda. ${ }^{12}$

Al analizar los datos obtenidos se observó, en común, que el estrés contribuye en forma significativa al aumento de la presencia y severidad del dolor en la espalda $(\mathrm{p}=<0.0001)$ y especialmente en el cuello $(p=0.042)$. Es probable que el dolor del cuello sea un problema más relevante dentro de la población, que el dolor lumbar en la categoría de moderado y de moderado a severo, ya que así lo muestran los porcentajes arrojados en los resultados.

Otros estudios han establecido como factores de riesgo las prácticas posturales inadecuadas, la debilidad muscular, el estrés y la edad; ${ }^{6}$ este estudio no encontró asociaciones estadísticamente significativas para dolor lumbar; ni en otras causas como: Comodidad de la silla, variación de la postura, objetos al alcance, tiempo laboral y dificultad para trabajar. Existen estudios realizados por Horstman y colaboradores, ${ }^{13} \mathrm{Al}$ Wazzan y colaboradores ${ }^{6}$ y Levangie ${ }^{14}$ que demostraron que estos son factores asociados al dolor lumbar y del cuello.

Consideradas las limitaciones de este estudio, se encontró que el planteamiento de las preguntas relacionadas con posibles causas, probablemente no fue lo suficientemente claro, debido a la falta de instrucción por parte de los inves-

\begin{tabular}{|c|c|c|c|}
\hline & Antes & Actualidad & $\mathbf{p}$ \\
\hline Presencia de dalor lumbar & $24 / 109$ & $74 / 109$ & $<0,0001$ \\
\hline Presencia de dolor de cuello & $17 / 109$ & 57109 & $<0.0001$ \\
\hline \multicolumn{4}{|l|}{ Dolor lumbar por semestre } \\
\hline 6 & $6 / 27$ & $17 / 30$ & $<0,0001$ \\
\hline 7 & $3 / 19$ & $13 / 19$ & 0.0016 \\
\hline 8 & $6 / 21$ & $13 / 21$ & 0.0082 \\
\hline 9 & $7 / 21$ & $16 / 21$ & 0.0027 \\
\hline 10 & $2 / 21$ & $15 / 21$ & 0.0008 \\
\hline \multicolumn{4}{|l|}{ Dolor de cuelle por semestre } \\
\hline 6 & $5 / 27$ & $13 / 29$ & 0.01 \\
\hline 7 & $1 / 19$ & $8 / 19$ & 0.082 \\
\hline 8 & $5 / 21$ & $9 / 21$ & 0.04 \\
\hline 9 & $3 / 21$ & $12 / 21$ & 0.0027 \\
\hline 10 & $3 / 21$ & $15 / 21$ & 0.0005 \\
\hline
\end{tabular}

Tabla 7. Comparación de la presencia de dolor antes y después del ingreso a la clínica. 
tigadores o a la falta de seriedad en el diligenciamiento del cuestionario, por los estudiantes de la muestra; probablemente, por ello no se pudieron establecer las asociaciones ya demostradas en otros trabajos. ${ }^{6,15}$

En la revisión de la literatura se encontró que en un estudio realizado en Nebraska, el $47 \%$ de más de mil odontólogos registraron tener síndrome del Túnel Carpiano, el cual hace parte de los desórdenes músculoesqueléticos y de las alteraciones funcionales, que se presentan frecuentemente en los odontólogos. ${ }^{16}$

El síndrome del túnel carpiano es una alteración que ocurre en alrededor del 48\% de los odontólogos encuestados en 1990; por esta razón es importante su valoracion en estudios posteriores. ${ }^{17}$ En este trabajo no se consideró la valoración de la presencia de este síndrome, lo que constituye otra limitación.

Una de las ventajas de este trabajo es, sin embargo, que los instrumentos utilizados para determinar la discapacidad o limitación funcional derivada del dolor del cuello y espalda, son instrumentos validados. ${ }^{8}$ En la literatura revisada, ninguno de los artículos en los cuales se consideró la valoración de estos aspectos en la población de odontólogos, utilizó instrumentos validados para determinar esta condición. ${ }^{18}$

En el estudio realizado por Caillard y colaboradores, se consideró la importancia de valorar este tipo de alteraciones de origen ocupacional, con el fin de implementar programas preventivos y mejorar los ya existentes, teniendo en cuenta los factores psicosociales, la organización laboral y la evaluación científica. ${ }^{19}$

Es importante resaltar, que este es el primer trabajo de investigación que se realiza en estudiantes de odontología relacionado con la salud ocupacional en la Universidad Santo Tomas, lo que conlleva la urgente necesidad formular programas de promoción de hábitos y posturas saludables en dicha población, y crear programas de prevención primaria, enfocados a disminuir las alteraciones del cuello, espalda y miembros superiores en estudiantes y futuros profesionales de la odontología.

\section{BIBLIOGRAFÍA}

1. Dagnino J. Definiciones y Clasificaciones del Dolor. Boletín Esc. de Medicina, P. Universidad Católica de Chile 1994; 23: 148 - 151. URL disponible en: http:// escuela.med.pub.cl/publicaciones/Boletín/html/ dolor/3_2.html
2. Chalen F, Escandon J. Medicina Interna. Fundación Instituto de Reumatología e Inmunología. Bogotá, 1998, Vol. II. p. 1940-1960.

3. Kottke J, lehmann JF. Medicina Física y Rehabilitación. 3 ed. Argentina, Editorial Interamericana. 1985. p: 124-136.

4. Carrillo JS. Ergonomía en Odontología: Planteamiento de necesidades. Profesión Dental. Revista científica y de información profesional 2001; 4: $227-235$.

5. Buckle M. GeoffD. Prevención de los trastornos musculoesqueleticos: Hacia un planteamiento global. Agency for Safety and Health at Work. 1998. p: 1-6.

6. Alwazzan K. Back \& Neck problems among dentists and dental auxiliaries. J Contemp Dent Pract 2001; 2: 1 - 9.

7. Praemer A, Rice DP. Musculoskeletal conditions in the United States. Rosemont: AAUS, 1992.p. 1-99.

8. Fair JC, Davies JB. The oswestry low back pain disability questionnaire. Physiotherapy 1980; 66: 271 - 273. URL disponible en: http:// www.medal.org/sheets/ch37/low\%20back\%200swestry\%

9. Pagano M, Gauvreau K. Principles of biostatistics. Duxbury Press, Belmont Ca, 1993. p: 11-12.

10. Microsoft Excel, 5.0 Microsoft corporations, 1997.-Stata Corp. 1999.Stata statistical

11. Software: Release 6.0. College station, Tx: Stata corporation.

12. Gale E. Stress in Dentistry. N Y State Dent J 1998; 8: 30 - 35.

13. Horstman SW, Horstman BC. Ergonomic risk factors associated with the practice of dental hygiene: A preliminary study. American Society of Safety Engineers [Online] 1997; 64: 49 - 53. URL disponible en: http://proquest.umi.com/pqdweb

14. Levangie PK. Association of low back pain with self - reported risk factors among patients seeking physical therapy services. Physical Therapy 1999; 79: 757 - 766. URL disponible en: http:// proquest.umi.com/pqdweb

15. Cole DC, Eyles F. Work correlates of back problems and activity. Restriction due to musculoskeletal disorders in the Canadian national population health survey (NPHS). Occup Envirom 1994; 58: 728 - 734.

16. Dale RF, Moriss - Allen MS. Musculoskeletal disorders in dentists. N Y State Dent J 1998; 64: 23 - 29.

17. Anonymous. New laser speeds therapy for repetitive stress injuries. Health Care Financing Review [Online] 1992; 14: 1 - 7 .

18. Steele S. Vertical anthropometric measures and low back pain in adolescent. Physiotherapy Research International 2001; 94 - 105.

19. Caillard JF, Iwatsubo Y. Prevention of musculoskeletal disorders among health care workers. Service Central de Medicine du Travail. Assistance Publique [Online] 2000:1 - 5. 